\title{
Outcome of pregnancy in women with congenital shunt lesions
}

\author{
M Zuber, N Gautschi, E Oechslin, V Widmer, W Kiowski, R Jenni
}

\begin{abstract}
Objective-To evaluate the outcome of pregnancy in women with congenital shunt lesions.

Setting-Retrospective study in a tertiary care centre.

Methods-Pregnancy history was obtained by a standardised questionnaire and medical records were reviewed.

Patients-175 women were identified, at a mean (SD) age of 42 (14) years. Pregnancies occurred in 126 women: 50 with an atrial septal defect, 22 with a ventricular septal defect, 22 with an atrioventricular septal defect, 19 with tetralogy of Fallot, and 13 with other complex shunt lesions. Results-309 pregnancies were reported by 126 woman $(2.5(1.6)$ pregnancies per woman). The shortening fraction of the systemic ventricle was $40(8) \%$, and $98 \%$ were in New York Heart Association class I-II at last follow up. Spontaneous abortions occurred in $17 \%$ of pregnancies (abortion rate, 0.4 (0.9) per woman). Gestational age of the 241 newborn infants was $8.8(0.8)$ months. There were no maternal deaths related to pregnancy. Pre-eclampsia and embolic events were observed in $1.3 \%$ and $0.6 \%$, respectively of all pregnancies. Women with complex shunt lesions more often underwent caesarean section $(70 \% v 15-30 \%, p=0.005)$ and gave birth to smaller babies for equivalent gestation (2577 (671) g $v 3016$ (572) to 3207 (610) g, p < 0.05). The recurrence risk of congenital heart disease was $2.5 \%$. Conclusions-The outcome of pregnancy is favourable in women with congenital shunt lesions if their functional class and their systolic ventricular function are good. Such patients can be reassured.

(Heart 1999;81:271-275)
\end{abstract}

Keywords: congenital heart defects; pregnancy

Division of

Echocardiography, University Hospital, Raemistrasse 100,

CH-8091 Zurich, Switzerland $M$ Zuber

N Gautschi

E Oechslin

V Widmer

W Kiowski

R Jenni

Correspondence to: Dr Jenni.

Accepted for publication 30 November 1998

especially in patients with cyanotic congenit heart disease, although the prognosis is improving. ${ }^{1-5}$ A recent study from Toronto reported that poor maternal functional class or cyanosis were associated with adverse neonatal events, ${ }^{6}$ and the management of pregnant women with congenital shunt lesions remains a problem. Our aim in this study was to evaluate the outcome of pregnancy and delivery in such women, to describe the complications of the early postpartum period and symptoms occurring during late follow up, and to identify the recurrence risk for congenital heart defects in the offspring.

\section{Methods}

STUDY POPULATION

We identified 217 patients in our supraregional referral and tertiary care centre database who had congenital shunt defects and were more than 18 years old (mean (SD) age, 42 (14) years). Ten patients were lost to follow up. The other 207 patients were contacted, but there was no response from 32 of them. Thus 175 women and their family physicians completed a standardised questionnaire. Of these, 126 reported pregnancies (mean age 45 (13) years): 50 had a secundum atrial septal or sinus venosus defect (surgically closed in 28 without residual shunt; one patient had moderate and one severe mitral regurgitation); 22 had a ventricular septal defect (surgically closed in 12 without residual shunt; four had mild to moderate right ventricular outflow tract obstruction with a peak gradient $<50 \mathrm{~mm} \mathrm{Hg}$ in all cases); 22 had a partial or total atrioventricular septal defect (corrective repair in 17; one had mild left ventricular outflow tract obstruction with a peak gradient of $20 \mathrm{~mm} \mathrm{Hg}$ ); 19 had tetralogy of Fallot (complete repair in all; none had haemodynamically important residual defects); and 13 had complex shunt lesions (definitive surgery in eight, palliative surgery in one, no surgery in four). Complex shunt lesions comprised truncus arteriosus (1), d-transposition of the great arteries (5), congenitally corrected transposition of the great arteries (3), univentricular connection (2), and pulmonary atresia with ventricular septal defect (2). In these 13 patients with complex lesions, cyanosis was present in three of four women who had not had surgery and in one who had had a Waterston anastomosis; eight women who had had definitive surgery were pink. Cyanosis was defined as the presence of central cyanosis with an underlying anatomy compatible with systemic arterial systemic desaturation. ${ }^{7}$

The study population thus consisted of 126 women who reported pregnancies, 84 of whom had undergone corrective or definitive surgery.

A standardised questionnaire was completed by the patients and their family physicians (general practitioners), using obstetric reports to obtain the pregnancy history, complications in the early postpartum period, and functional 
Table 1 Characteristics of 126 women with congenital shunt lesions and a history of pregnancy

\begin{tabular}{llllll}
\hline & ASD & VSD & Fallot & AVSD & Complex \\
\hline Patients (n) & 50 & 22 & 19 & 22 & 13 \\
Corrective surgery (n (\%)) & $28(56)$ & $12(55)$ & $19(100)^{\star}$ & $17(77)$ & $8(62)$ \\
Age at corrective surgery (years) & $37(19) \dagger$ & $21(19)$ & $18(12)$ & $20(11)$ & $12(17)$ \\
Age at last follow up (years) & $48(14)$ & $47(13)$ & $40(10)$ & $43(9)$ & $38(13)$ \\
Left to right shunt (\%) & $48(15)$ & $41(16)$ & $43(13)$ & $49(15)$ & No data \\
Shortening fraction (\%) $\ddagger$ & $40(7)$ & $37(7)$ & $36(8)$ & $43(10)$ & -
\end{tabular}

Values are means (SD) unless otherwise stated.

${ }^{\star} \mathrm{p}<0.01$, Fallot $v$ ASD/VSD/complex.

tp $=0.0003$, ASD $v$ Fallot.

$\ddagger$ Shortening fraction of the systemic ventricle.

ASD, atrial septal defect; AVSD, atrioventricular septal defect; Complex, complex shunt lesions; Fallot, tetralogy of Fallot; VSD, ventricular septal defect.

status during late follow up. In Switzerland, deliveries are performed by gynaecologists who provide an obstetric report to the family physicians. The principal anatomical diagnosis, surgical history, and haemodynamic data were obtained from the women's medical records.

A miscarriage was defined as spontaneous fetal loss before 20 weeks of gestation. Pregnancies ending in therapeutic abortions were not included. Pregnancies were analysed separately in each diagnostic group and compared between groups.

The shortening fraction of the systemic ventricle (end diastolic diameter minus end systolic diameter divided by end diastolic diameter $\times$ 100) was used for quantitative evaluation of systemic ventricular function. The ventricular dimensions were measured using cross sectionally guided $M$ mode echocardiography in the parasternal long axis view. Cardiac output was measured by the Fick method and the percentage left to right shunt was calculated (from the oxygen saturation) or determined noninvasively by Doppler echocardiography before surgical repair, or at the time of the last follow up visit in patients without corrective surgery. ${ }^{8}$

The numbers of pregnancies before and after corrective surgery were obtained and any complications recorded. Complications included pre-eclampsia and thromboembolic events. Pre-eclampsia was defined as systemic hypertension, oedema, and proteinuria requiring medical attention. General wellbeing (subjective evaluation by the woman) and functional class (New York Heart Association (NYHA) class) were assessed during late follow up.
STATISTICAL ANALYSIS

All data are expressed as mean (SD). Categorical variables were tested with $\chi^{2}$ analysis of variance and Fisher's PLSD test at 95\% significance level. Analysis of variance was used for comparison of continuous variables between groups, with Bonferroni correction if the analysis indicated overall significance between groups. A p value $<0.05$ was considered statistically significant.

\section{Results}

Fifty women with an atrial septal defect, 22 with a ventricular septal defect, 19 with tetralogy of Fallot, 22 with an atrioventricular septal defect, and 13 with complex shunt defects reported pregnancies. Mean age at last follow up of the 126 patients who had become pregnant was 45 (13) years; $84(67 \%)$ of them underwent corrective or definitive surgery at a mean age of 24.4 (18) years. The mean shortening fraction was $40(8) \%$ and cyanosis was present in $2 \%$.

Table 1 presents the characteristics of all the women who had been pregnant, in their different diagnostic groups. All women with tetralogy of Fallot had undergone corrective surgery; in that respect, this group differed significantly $(p<0.01)$ from those with atrial septal defect, ventricular septal defect, and complex lesions. Women with an atrial septal defect were older at the time of corrective surgery than those with tetralogy of Fallot $(p=0.0003)$. The left to right shunt and the shortening fraction of the systemic ventricle were comparable between the different diagnostic groups. The shortening fraction of the systemic ventricle was normal in all patients at late follow up.

Ejection fraction was determined invasively in 10 patients with complex lesions. In three of five women who had not undergone corrective surgery the value was less than $60 \%$; in five patients who had undergone corrective surgery, the calculated ejection fraction was greater than $60 \%$. Angiographic determination of ejection fraction was not done in three patients.

Overall, 309 pregnancies were reported by the 126 women, giving a mean (SD) pregnancy rate of 2.5 (1.6) per woman; mean pregnancy duration was $8.8(0.8)$ months and the mean

Table 2 Characteristics of 309 pregnancies in 126 women with congenital shunt lesions

\begin{tabular}{llllll}
\hline & ASD & VSD & Fallot & AVSD & Complex \\
\hline Number of patients & 50 & 22 & 19 & 22 & 13 \\
Pregnancies (n) & 126 & 63 & 44 & 48 & 28 \\
Pregnancies/woman & $2.5(1.4)$ & $2.9(2.0)$ & $2.3(1.5)$ & $2.3(1.4)$ & $2.0(1.8)$ \\
Women with surgery & & & & \\
$\quad$ Pregnancies before surgery/woman & $0.6(1.4)^{\star}$ & $0.2(0.5)$ & $0.5(1.1)$ & $0.1(0.3)$ & $0.1(0.6)$ \\
$\quad$ Pregnancies after surgery/woman & $1.6(1.2)$ & $2.6(2.2)$ & $1.6(0.9)$ & $2.1(1.5)$ & $1.8(1.7)$ \\
Abortion rate (\%) & 16 & 10 & 16 & 17 & 36 \\
Caesarean section rate (\%) & 15 & 15 & 29 & 30 & $70 \dagger$ \\
Pregnancy duration (months) & $8.8(0.8)$ & $9.0(0.2)$ & $9.0(0.4)$ & $8.9(0.5)$ & $8.2(1.8)$ \\
Pre-eclampsia/embolisation (n) & $2 / 1$ & $1 / 1$ & $0 / 0$ & $1 / 0$ & $0 / 0$ \\
Reduced wellbeing (\%) & 10 & 10 & 24 & 5 & 22 \\
NYHA class I-II at last follow up (\%) & 100 & 100 & 95 & 100 & $85 \ddagger$
\end{tabular}

Values are means (SD) unless otherwise stated.

${ }^{\star} \mathrm{p}=0.05$, ASD $v$ VSD $/$ AVSD and complex; $\nmid \mathrm{p}=0.005$, complex $v$ ASD, VSD, Fallot, AVSD; $\neq \mathrm{p}=0.005$, complex $v$ ASD ASD, atrial septal defect; AVSD, atrioventricular septal defect; Complex, complex shunt lesions; Fallot, tetralogy of Fallot; NYHA, New York Heart Association; reduced wellbeing, worse wellbeing at the time of last follow up than before pregnancy; VSD, ventricular septal defect. 
Table 3 Neonatal data in 241 offspring by diagnostic group

\begin{tabular}{llllll}
\hline & ASD & VSD & Fallot & AVSD & Complex \\
\hline Offspring (n) & 102 & 52 & 31 & 39 & 17 \\
Crown-heel length (cm) & $49(3)$ & $49(2)$ & $49(3)$ & $49(2)$ & $46(5)^{\star}$ \\
Birth weight (g) & $3207(610)$ & $3181(291)$ & $3016(572)$ & $3090(533)$ & $2577(671)^{\star}$ \\
$\quad<2500$ g (n (\%)) & $5(5)$ & $0(0)$ & $3(10)$ & $2(5)$ & $4(24)^{\star}$ \\
Recurrence of CHD (n (\%)) & $4(3.9)$ & $1(1.9)$ & 0 & $1(2.6)$ & 0 \\
\hline
\end{tabular}

Values are mean (SD) unless otherwise stated.

${ }^{\star} \mathrm{p}<0.05$, complex $v$ all other groups.

ASD, atrial septal defect; AVSD, atrioventricular septal defect; CHD, congenital heart disease per live birth; Complex, complex shunt lesions; Fallot, tetralogy of Fallot; VSD, ventricular septal defect.

miscarriage rate (spontaneous fetal loss) was 0.4 (0.9) per woman (51 abortions; $17 \%$ ). Table 2 gives the characteristics of these 309 pregnancies. The number of pregnancies, rate of complications, and incidence of reduction in wellbeing at the time of the last follow up visit were comparable among the groups, but there was a trend towards a higher abortion rate in patients with complex lesions. However, in women with an atrial septal defect more pregnancies occurred before corrective repair than in the other groups, because in many cases the diagnosis had not been made before these women became pregnant. This explains why they were older at the time of corrective surgery (table 1).

Pre-eclampsia and embolic events were observed in $1.3 \%$ and $0.6 \%$ of all pregnancies, respectively. Embolic events included pulmonary embolism in one woman and a cerebrovascular accident in another. The duration of pregnancy was slightly shorter in patients with complex shunt lesions, who were more often delivered by caesarean section $(p=0.005)$. At the time of the last follow up, patients with complex shunt lesions reported that they were more dyspnoeic than those with atrial septal defects $(p=0.005)$. Cardiac reasons were never cited as an indication for caesarean section.

Two thirds of the patients $(n=84 ; 67 \%$ ) underwent corrective or definitive surgery. As expected, women who had undergone corrective/definitive surgery had significantly larger left to right shunts preoperatively (52 $(12) \%$ v $36(15) \%)$, and were not cyanotic $(0 \%$ $v 7.3 \%)$ at follow up. The number of pregnancies per woman $(2.6(1.8) v 2.2(0.9))$ was not statistically different between women with and without surgery. Mean age at last follow up (45 (12) $v 45$ (14) years), shortening fraction of the systemic ventricle (40 (8)\% v 39 (8)\%), duration of pregnancy $(8.8 \quad(0.8) \quad v \quad 8.8 \quad(0.9)$ months), number of live births per woman (2.0 $(1.3) v 2.0(0.8)$ ), abortion rate $(18 \% v 13 \%)$, caesarean section rate $(24 \% v 26 \%)$, and complication rate during pregnancy (pre-eclampsia $4.7 \%$ v $0 \%$; embolic events $1.2 \%$ v $2.4 \%$ ) were comparable between women with and without corrective/definitive surgery. Birth weight (3121 (549) $v 3057$ (613) g) and body length (49 (3) $v 48(4) \mathrm{cm}$ ) were also not statistically different between these two groups.

Only one woman reported having arrhythmias during pregnancy. This patient had transposition of the great arteries and had undergone the Mustard procedure. The ar- rhythmias were successfully treated with $\beta$ blockers. No patient developed heart failure during pregnancy or in the postpartum period.

Thirteen of the 24 women with complex lesions had 28 pregnancies (2.2 (1.7) pregnancies per woman). These 13 patients gave birth to 17 offspring, among whom there were no reported cases of congenital heart defects.

There were 241 completed pregnancies in the series. Body size and birth weight of the offspring were comparable between the different diagnostic groups (table 3). However, the babies of mothers with complex shunt lesions were slightly, but significantly, smaller than those of mothers with other defects.

The four cyanotic patients had five live births. One baby died on the second day after delivery. This baby, whose mother had pulmonary atresia and a ventricular septal defect, was delivered by caesarean section for impending asphyxia in the seventh month of gestation. The cause of death was unknown.

The risk of recurrent congenital heart defects in the 241 completed pregnancies was $2.5 \%$ (table 3 ). No heart defects were found in the offspring of mothers with tetralogy of Fallot or complex shunt lesions. Most defects were found in offspring of mothers with atrial septal defects, with the same lesion predominating in these babies (three with an atrial septal defect and one with a ventricular septal defect were born alive; one with a complex congenital heart defect was spontaneously aborted in the 20th week of gestation). One woman with a ventricular septal defect gave birth to a baby with a ventricular septal defect. One woman with an atrioventricular septal defect gave birth to a baby with a complex lesion.

Overall mortality in the women who had become pregnant was $1.6 \%(n=2$; one with an atrial septal defect and one with an atrioventricular septal defect). Both deaths occurred more than one year after delivery.

\section{Discussion}

People with congenital heart disease are increasingly reaching adulthood owing to remarkable diagnostic and therapeutic advances in cardiology and cardiac surgery. ${ }^{1}$ Thus there are now many women with congenital heart disease who are considering pregnancy, and the maternal and fetal risks in this heterogeneous group of patients must be carefully evaluated. In this retrospective study we report the experience of a single centre relating to the outcome of pregnancy in women with congenital shunt lesions, including atrial and ventricular septal defects, tetralogy of Fallot, atrioventricular septal defects, and other complex shunt lesions.

The pregnancy rate (2.5 per woman) and birth rate (2.0 children per woman) in our patients were higher than those in the general population in Switzerland, where the birth rate is 1.5 per woman. ${ }^{9}$ Only 0.5 pregnancies per woman were observed before corrective surgery, though women with an atrial septal defect had more pregnancies before surgery than women in the other diagnostic groups (table 2). Many of these women did not know they had a 
heart defect before they became pregnant. This observation explains their greater age at the time of corrective surgery (table 1). The overall outcome of pregnancy was good in our population.

The outcome of pregnancy was not associated with the type of previous surgery. This is consistent with the findings in a prospective study of 233 women with congenital heart disease and a recently published retrospective study of a further 221 women. ${ }^{6}{ }^{10}$ Poor maternal functional class or cyanosis were independent predictors of neonatal events. ${ }^{6}$ The abortion rate was 0.4 per woman $(17 \%)$, which is higher than previously reported. ${ }^{11}$

The duration of pregnancy was shorter in women with complex lesions, who gave birth to smaller babies for equivalent gestation than the women in the other groups. However, neither the abortion rate nor the complication rate differed statistically between the diagnostic groups, though there was a trend towards a higher abortion rate in women with complex lesions. The shorter duration of pregnancy in women with complex lesions can be explained by the fact that they more often had elective caesarean sections ( $70 \%$ v 15-30\%). Although heart problems were not cited as the reason for caesarean section in any of these women, the complex anatomy may have led to the higher caesarean section rate in this population.

Shime et al reported a major benefit of corrective cardiac surgery in cyanotic patients, leading to fewer spontaneous abortions and more term births. ${ }^{12}$ Our four cyanotic patients had five live births, but one child died on the second day. The risk of pregnancy in cyanotic patients without pulmonary hypertension has been shown to be low for the mother, but the fetal outcome is poor, with high rates of abortion, intrauterine growth retardation, intraventricular haemorrhage, premature birth, and neonatal death. ${ }^{7}$ A recently published retrospective study that did not include women with Eisenmenger syndrome did not confirm an association between pulmonary hypertension and pregnancy complications. ${ }^{6}$ However, this study only included patients with moderate pulmonary hypertension, who are at lower risk than patients with Eisenmenger syndrome. Careful monitoring in the intensive care unit is recommended for the most favourable outcome in patients with cyanosis and pulmonary artery hypertension. ${ }^{13-16}$

Pregnancies in women who have had an atrial switch operation or a Fontan procedure are reported to be well tolerated in selected groups, but the incidence of abortion is high during the first trimester. ${ }^{17-19}$ A Senning procedure was performed in three of our patients and a Mustard procedure in two, but no women underwent a Fontan procedure in our population.

Pregnancy and its outcome were comparable among the different diagnostic groups, excluding those with complex shunt lesions. This observation is consistent with other reports. ${ }^{2021}$ In our study, patients with complex lesions were more often symptomatic and complained of more dyspnoea than the others during late follow up (table 2). The worse functional status in these patients (reflected by their NYHA class) may be explained by their more complex anatomical abnormalities and their residual haemodynamic abnormalities.

The incidence of pre-eclampsia in our patients was comparable with that in the general population. ${ }^{22}$

Embolic events are reported to occur in $0.7 \%$ of healthy individuals during pregnancy and the risk seems to be increased in the postpartum period. ${ }^{23}{ }^{24}$ This observation is similar to that in our population $(0.6 \%)$ including only a small number of women with complex lesions and cyanosis. However, patients with cyanotic congenital heart defects are reported to be at higher risk of embolic events. ${ }^{25}$

Long term mortality was $1.6 \%$ in our patients who had reported pregnancies. However, not all deaths were related to pregnancy or delivery.

The recurrence risk of congenital heart disease was $2.5 \%$ and was not affected by the principal diagnosis. The recurrence rate was highest in women with atrial septal defects and the same lesion predominated in their offspring. The fetal risk of recurrence of congenital heart disease is reported to be $0 \%$ to $11 \%{ }^{26-28}$ No routine echocardiographic assessment was performed in the newborn infants. Thus we may have underestimated the recurrence rate in our population. However, most newborn infants are followed by a paediatrician and all children undergo regular medical examinations at school in Switzerland. Thus the number of missed diagnoses is likely to be low. The prevalence of small for dates babies in women with atrial septal defects, ventricular septal defects, and atrioventricular septal defects was comparable with that in an unselected US population $(6 \%$ of babies $<2500 \mathrm{~g}$ at term), ${ }^{11}$ but it was much higher in our patients with tetralogy of Fallot and complex shunt lesions.

LIMITATIONS OF THE STUDY

The population of this retrospective study, performed at a tertiary care centre, may be highly selected. Furthermore, there may be a bias because of the $19.4 \%$ exclusion rate (10 patients lost to follow up; no response from 32 patients). There was incomplete information about the outcome (abortion, completed pregnancy) in $5.5 \%$ of pregnancies.

Selection bias was minimised by the high referral rate of patients with congenital heart disease to our division of echocardiography. Data collection bias was minimised by the use of a standardised questionnaire. The questionnaires administered to patients and family physicians may yield incomplete information because in many cases the events occurred years ago. In Switzerland, however, an obstetric report is provided to the general practitioners who care for the patients after delivery. Thus bias reflecting incomplete information is considered to be minimal. The complication rate in our population may be underestimated because of the retrospective study design and the lack of a consistent diagnostic strategy for 
identifying complications (for example, scanning for intraventricular haemorrhage). As this study was a retrospective design, risk factors for complications were not determined.

CONCLUSIONS

The outcome of pregnancy is favourable in women with congenital shunt lesions and is comparable between those with atrial and ventricular septal defects, atrioventricular septal defects, tetralogy of Fallot, and complex lesions, if their functional class and systemic ventricular function are good. Maternal and fetal morbidity and long term outcome are acceptable, and there were no maternal deaths related to pregnancy or delivery. This group of women and their partners can thus be reassured.

Women with complex shunt lesions, including those with palliative and corrective/ definitive surgery, or those with simple shunt lesions (atrial and ventricular septal defects) who have residual shunts after corrective surgery or who have long term complications irrespective of their functional class, must be assessed by a team including an obstetrician skilled in high risk pregnancies and a cardiologist with special expertise in this group of patients to anticipate potential maternal and fetal complications. The low fetal recurrence rate of congenital heart disease $(2.5 \%)$ does not preclude pregnancy.

Preconception management should, if possible, include corrective repair of haemodynamically significant shunt lesions or palliation to alleviate cyanosis and to reduce both maternal and fetal risk. Postconception counselling should offer fetal echocardiography performed by a skilled sonographer to identify possible genetic transmission of heart defects. A prospective study including age matched women as controls is needed to identify risk factors for an unfavourable outcome.

1 Perloff JK. Pregnancy and congenital heart disease. $7 \mathrm{Am}$ Coll Cardiol 1991;18:340-2.

2 Pitkin RM, Perloff JK, Koos BJ, et al. Pregnancy and congenital heart disease. Ann Intern Med 1990;112:445-54

3 Somerville J. Congenital heart disease in adults and adolescents. Br Heart $\mathcal{F}$ 1986;56:395-7.

4 Wooley CF, Sparks EH. Congenital heart disease, heritable Wooley CF, Sparks EH. Congenital heart disease, heritable cardiovascular dise

5 Sullivan JM, Ramanathan KB. Management of medical problems in pregnancy - severe cardiac disease. $N$ Engl $\mathscr{F}$ Med 1985;313:304-9.
6 Siu SC, Sermer M, Harrison DA, et al. Risk and predictors for pregnancy-related complications in women with heart disease. Circulation 1997;96:2789-94.

7 Presbitero P, Somerville J, Stone S, et al. Pregnancy in cyanotic congenital heart disease. Outcome of mother and fetus. Circulation 1994;89:2673-6.

8 Jenni R, Ritter M, Vieli A, et al. Determination of the ratio of pulmonary blood flow to systemic blood flow by derivation of amplitude-weighted mean velocity from continuous wave Doppler spectra. Br Heart 7 1989;61:167-71.

9 Bundesamt fuer Statistik, 1997. Lebengeborene Kinder 1950-1995. In: Statistisches fahrbuch der Schweiz 1997. Zurich: Verlag Neue Zuercher Zeitung, 1997:45.

10 Whittemore R, Hobbins JC, Engle MA. Pregnancy and its outcome in women with and without surgical treatment of congenital heart disease. Am f Cardiol 1982;50:641-51.

11 Reiter RC. The health of women: a current perspective. In: Moore TR, Reiter RC, Rebar RW, et al, eds. Gynecology and obstetrics: a longitudinal approach. New York: Churchill Livingstone, 1993:1-9.

12 Shime J, Mocarski EJ, Hastings D, et al. Congenital heart disease in pregnancy: short- and long-term implications. Am $\mathcal{F}$ Obstet Gynecol 1987;156:313-22.

13 Alon E, Baumann H. Anesthesiologic management of cesarean section in a patient with transposition of the great vessels. Reg Anesth 1988;11:28-31.

14 Weiss BM, Atanassoff PG. Cyanotic congenital heart disease and pregnancy: natural selection, pulmonary hypertension, and anesthesia. F Clin Anesth 1993;5:332-41.

15 Atanassoff PG, Schmid ER, Jenni R, et al. Epidural anesthesia for a cesarean section in a patient with pulmonary atresia and ventricular septal defect. F Clin Anesth 1991;3:399-402.

16 Spinnato JA, Kraynack BJ, Cooper MW. Eisenmenger's syndrome in pregnancy: epidural anesthesia for elective cesarean section. N Engl f Med 1981;304:1215-17.

17 Clarkson PM, Wilson NJ, Neutze JM, et al. Outcome of pregnancy after the Mustard operation for transposition of the great arteries with intact ventricular septum. $\mathcal{F} \mathrm{Am}$ Coll Cardiol 1994;24:190-3.

18 Lao TT, Sermer M, Colman JM. Pregnancy following surgical correction for transposition of the great arteries. Obstet Gynecol 1994;83:665-8.

19 Canobbio MM, Mair DD, van der Velde M, et al. Pregnancy outcomes after the Fontan repair. F Am Coll Cardiol 1996; 28:763-7.

20 Singh H, Bolton PJ, Oakley CM. Pregnancy after surgical correction of tetralogy of Fallot. Br Med f Clin Res $1982 ; 285: 168-70$

21 Hess DB, Hess LW. Management of cardiovascular disease in pregnancy. Obstet Gynecol Clin North Am 1992;19:6795

22 Repke JT. Hypertension and preeclampsia. In: Moore TR, Reiter RC, Rebar RW, et al, eds. Gynecology and obstetrics: a longitudinal approach. New York: Churchill Livingstone, 1993:463-77.

23 O'Brien W. Puerperal complications. In: Moore TR, Reiter $\mathrm{RC}$, Rebar RW, et al, eds. Gynecology and obstetrics: a longi-
tudinal approach. New York: Churchill Livingstone, 1993: tudinal appro $637-54$.

24 Kittner SJ, Stern BJ, Feeser BR, et al. Pregnancy and the risk of stroke. N Engl F Med 1996;335:768-74.

25 Ammash N, Warnes CA. Cerebrovascular events in adult patients with cyanotic congenital heart disease. $f \mathrm{Am}$ Coll Cardiol 1996;28:768-72.

26 Nora JJ, Nora AH. Maternal transmission of congenital heart diseases: new recurrence risk figures and the questions of cytoplasmic inheritance and vulnerability to teratogens. Am F Cardiol 1987;59:459-63.

27 Rose V, Gold RJ, Lindsay G, et al. A possible increase in the incidence of congenital heart defects among the offspring of affected parents. 7 Am Coll Cardiol 1985;6:376-82.

28 Whittemore R, Wells JA, Castellsague X. A secondgeneration study of 427 probands with congenital heart generation study of 427 probands with congenital heart
defects and their 837 children. $\mathcal{F}$ Am Coll Cardiol 1994;23: 1459-67. 